\title{
Changing Trends in Rate of Cervical Dilation in First Stage of Labor: Prospective Longitudinal Study
}

\author{
Divyesh V. Shukla1 ${ }^{*}$, Shilpi D. Shukla1, Sangita Patel ${ }^{2}$, Amit Shah ${ }^{1}$ \\ ${ }^{1}$ Isha Hospital, Vadodara, India \\ ${ }^{2}$ Department of PSM, Medical College Baroda, Vadodara, India \\ Email: *divyeshshukla@yahoo.com
}

How to cite this paper: Shukla, D.V., Shukla, S.D., Patel, S. and Shah, A. (2020) Changing Trends in Rate of Cervical Dilation in First Stage of Labor: Prospective Longitudinal Study. Open Journal of $\mathrm{Ob}$ stetrics and Gynecology, 10, 1176-1186. https://doi.org/10.4236/ojog.2020.1090111

Received: July 20, 2020

Accepted: September 7, 2020

Published: September 10, 2020

Copyright $\odot 2020$ by author(s) and Scientific Research Publishing Inc. This work is licensed under the Creative Commons Attribution International License (CC BY 4.0).

http://creativecommons.org/licenses/by/4.0/

\begin{abstract}
Introduction: Knowledge of change in the duration of stages of labour would be an essential step to reduce the increasing rates of cesarean section reported worldwide. Objective: To study the rate of cervical dilation in the $1^{\text {st }}$ stage of labour in spontaneous and induced labour and in primigravida and multigravida with singleton pregnancy. Methods: A prospective observational study conducted at a multispeciality hospital was carried out for a period of 3 years from Jan 2017 to Dec 2019. A total of 640 patients who were admitted with spontaneous and induced labour having singleton pregnancy with cephalic presentation and intact membranes after 34 weeks who delivered vaginally were included for analysis. Progression of labor in the $1^{\text {st }}$ stage of labour was measured by the rate of cervical dilation as noted by serial per vaginal examination and findings were plotted in partograph. Result: The difference between mean rate of cervical dilation in the study group is statistically significant between $4-6 \mathrm{~cm}$ and $6-10 \mathrm{~cm}(\mathrm{P}<0.0001)$. When primigravida \& multigravida patients were compared for the cervical dilation rate, statistically significant difference was seen between $4-6 \mathrm{~cm}$ but not in $6-10 \mathrm{~cm}$. Average rate of cervical dilatation was 3.44 (Standard Deviation $(\mathrm{SD})=1.84$ ) in spontaneous labor while average rate of cervical dilatation was $2.69(\mathrm{SD}=1.18)$ in induced labor between $6-10 \mathrm{~cm}$ of cervical dilatation. Conclusion: The active phase of labour starts at $6 \mathrm{~cm}$ of cervical dilation in the majority of the patients. In multigravida, cervix dilates at faster rate before $6 \mathrm{~cm}$. In induced labour cervix dilates at a slower rate than spontaneous labour after $6 \mathrm{~cm}$ dilation.
\end{abstract}

\section{Keywords}

Cervical Dilatation, Spontaneous Labour, Induced Labour, Active Phase of Labour 


\section{Introduction}

It has been observed that the progress of labour in this century is not the same as criteria laid down by Friedman in 1955 and Philpott in 1972 [1] [2]. For the last 64 years we are following norms laid by Friedman to manage progress of labour and define abnormal labour. Many observational studies in recent times have reported changes in the duration of labour and rate of cervical dilation which is different from the criteria laid down by Friedman. This necessitates knowledge of change in the timings for confirming disorders of the progress of labour. This would be an essential step in context to reducing the increasing rates of cesarean section reported worldwide. Hence it is important to document the new normal limits of the duration of stages of labour, the rate of cervical dilation, and define abnormal labour in the current century. Worldwide many studies have reported this change. It has been observed that the rate of cervical dilation accelerated at 5 or $6 \mathrm{~cm}$ dilation. The cervical dilation rate from 4 to $6 \mathrm{~cm}$ was far slower than previously described. Hence women spent longer time in labour before $6 \mathrm{~cm}$ of cervical dilation. Knowledge of this fact may reduce the rate of primary and subsequent repeat cesarean deliveries [3] [4].

Study design-Prospective longitudinal observational study.

Objectives-To study the following in spontaneous and induced labour in primigravida and multigravida with singleton pregnancy and cephalic presentation delivered vaginally.

1) To assess the rate of cervical dilation in the 1st stage of labour between 4 - 6 $\mathrm{cm}$ and $6-10 \mathrm{~cm}$.

2) To find out when in the 1st stage of labour rapid cervical dilation begins in spontaneous and induced labour.

3) To compare the cervical dilation rate between primigravida and multigravida.

\section{Methods}

This was a prospective observational longitudinal study carried out for 3 years from Jan 2017 to Dec 2019 at Isha multispeciality hospital, Vadodara, India. The study was approved by the ethics committee of the hospital.

Inclusion criteria-The study group consisted of all registered patients admitted in labour room with spontaneous and induced labour having singleton pregnancy with cephalic presentation after 34 weeks of pregnancy for 3 years who delivered vaginally. A total of 1600 patients delivered during the study period. Out of these 960 patients were excluded from the study as per the exclusion criteria mentioned below. The data of 640 patients who delivered vaginally were included for analysis.

Exclusion criteria-

1) Patients delivered during the process of labour by cesarean section for various maternal or fetal indications.

2) Preterm delivery, maturity $<34$ complete weeks of pregnancy. 
3) Patients admitted with advanced spontaneous labour with or without ruptured membranes (patients who on admission had $>4 \mathrm{~cm}$ cervical dilation).

4) Multiple pregnancy and other than cephalic presentation.

5) Evaluation of the $2^{\text {nd }}$ stage labour duration was not a part of this study.

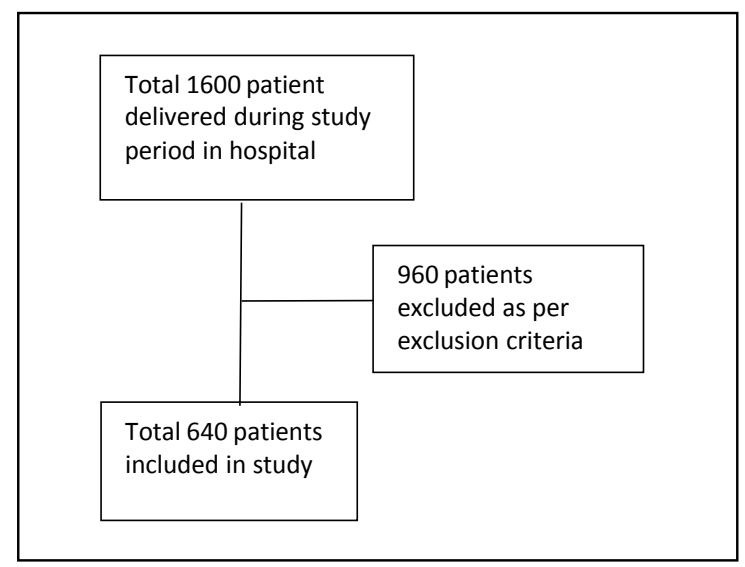

Informed consent was obtained from the patients. In antenatal period the hospital followed protocol to perform clinical pelvimetry after completion of 36 weeks. Cephalopelvic disproportion was ruled out again when they were admitted with labour pains. Bishop score was assessed at the time of admission for all patients. Vital signs of patients were monitored throughout the labour. In patients who were admitted for induction of labour [5] with intact membranes, induction was done by use of vaginal misoprostol 25 - 50 microgram which was repeated 4 - 6 hourly until cervical dilation had reached $4 \mathrm{~cm}$ and or effacement of about 50\% was achieved. Oral misoprostol in dose of 25 micrograms was used in patients who have Premature Rupture of Membranes (PROM) or Preterm Premature Rupture of Membranes (PPROM) at 4 hourly intervals [6] [7] [8]. Maximum dose of misoprostol used was 200 micrograms in 24 hours.

Active management of labour was practiced in all. Artificial rupture of membranes was done in patients with intact membranes when cervical dilation had reached $4 \mathrm{~cm}$ with effacement $50 \%$ or more. Station of fetal head was noted. Simultaneously oxytocin infusion was started by using infusion pump only at a rate of $2-4 \mathrm{miu} / \mathrm{ml} / \mathrm{min}$ in incremental fashion to attain adequate uterine contractions as per the stage of labour [9] [10].

Epiudural catheter was placed for patients who opted for labour analgesia early in labour and labour analgesia was begun after adequate uterine contraction began at approximate cervical dilation between $4-6 \mathrm{~cm}$. (As per standard protocol of epidural analgesia) [6] [11].

Progress of labour was assessed clinically by per abdomen examination and per vaginal examination noting cervical dilation \& station of head thereby assessing progress of labour by identified doctor and the trained nursing staff. Findings were plotted against the time spent in labour on WHO partogram. Fetal heart rate was monitored by using continuous CTG or intermittent ausculta- 
tion in all. Latent phase duration was calculated by noting time of initiation of mild uterine contractions (beginning of labour pain as communicated by patient) to time taken to reach $4 \mathrm{~cm}$ cervical dilation during the observation period in the hospital [12].

The data was entered in MS Excel sheet and analyzed using Medcalc software, Descriptive statistics such as mean and SD was calculated. Standard error of difference between two means ( $\mathrm{Z}$ test) was applied to see the statistical difference in the rate of cervical dilation between $4-6 \mathrm{~cm}$ and $6-10 \mathrm{~cm}$ of cervical dilatation, spontaneous and induced labour and primigravida and multigravida.

\section{Results}

Data analysis was carried out for 640 numbers of patients. The data shows usual patient distribution in childbearing age in the urban population in our city. In the study group primigravida (53.43\%) and multigravida (46.56\%) were almost equal in number. Similarly the number of patients with spontaneous onset of labour pains (57.96\%) and the patients who had induced labour (42.03\%) were also nearly the same in the study group. Associated medical conditions (pregnancy induced hypertension, gestational diabetes, hypothyroidism, anaemia) were present in a total of $114(17.81 \%)$ patients. Equal number of the patients were distributed between $<26$ or between $26-35 \mathrm{BMI}$ in the present study.

The current study and analysis do not aim to study the effect of these variables on duration of labour.

Bishop score was calculated for all, $512(80 \%)$ patients had favourable score between $6-13$ and the remaining $128(20 \%)$ had unfavourable score $<6$.

The socio-demographic characteristics of the patients are summarized in $\mathrm{Ta}$ ble 1 .

Labour analgesia in the form of epidural analgesia was opted by $2 \%$ of patients.

Latent phase duration of $<8$ hours was observed in $56.25 \%$ patients. Long latent phase duration up to 58 hours was observed in patients with induced labour ( 8 - 16 hrs. in $35.46 \%$ and $16-28$ hrs. in $8.28 \%$ and up to 58 hours in $0.31 \%$ ) (Table 2).

According to Table 3, difference between mean rate of cervical dilation in study group was statistically significant between $4-6 \mathrm{~cm}$ (mean $=1.71, \mathrm{SD}=$ 1.35) cervical dilation and $6-10 \mathrm{~cm}$ cervical dilation (mean $=3.14, \mathrm{SD}=1.64)(\mathrm{P}$ $<0.0001,95 \% \mathrm{CI}=1.19-1.66)$.

The average rate of cervical dilatation was $1.72 \mathrm{~cm}(\mathrm{SD}=1.10)$ in spontaneous labor and $1.68 \mathrm{~cm}(\mathrm{SD}=1.63)$ in induced labor between $4-6 \mathrm{~cm}$ of cervical dilatation at $95 \% \mathrm{CI}(-0.252$ to 0.172$)$. The difference was not statistically significant $(\mathrm{P}$ value $=0.711)$. The average rate of cervical dilatation was $3.44 \mathrm{~cm}(\mathrm{SD}=$ $1.84)$ in spontaneous labor and $2.69 \mathrm{~cm}(\mathrm{SD}=1.18)$ in induced labor between 6 $10 \mathrm{~cm}$ of cervical dilatation at $95 \%$ CI $(-1.001$ to -0.499$)$ which was statistically significant $(\mathrm{P} \leq 0.0001)$ as found in Table 4. 
Table 1. Patient socio-demographic characteristics.

\begin{tabular}{|c|c|}
\hline Socio-demographic characteristics & No. (\%) of patients \\
\hline \multicolumn{2}{|c|}{ Age } \\
\hline$\leq 30$ years & $492(76.87)$ \\
\hline$>30$ years & $148(23.12)$ \\
\hline \multicolumn{2}{|c|}{ Gravida } \\
\hline Primigravida & $342(53.43)$ \\
\hline Multigravida & $298(46.56)$ \\
\hline \multicolumn{2}{|l|}{ Onset of labour } \\
\hline Spontaneous & $371(57.96)$ \\
\hline Induced & $269(42.03)$ \\
\hline \multicolumn{2}{|l|}{ BMI } \\
\hline$<26$ & $320(50)$ \\
\hline$<26-35$ & $320(50)$ \\
\hline \multicolumn{2}{|l|}{$>35$} \\
\hline $\begin{array}{c}\text { Associated medical conditions PE, GD } \\
\text { Hypothyroidism, Anaemia }\end{array}$ & $114(17.81)$ \\
\hline Bishop score (6 - 13) & $512(80)$ \\
\hline
\end{tabular}

Table 2. Latent phase duration.

\begin{tabular}{ccccc}
\hline Latent phase & $<8$ hrs. & $8-16$ hrs. & $>16-28$ hrs. & $=58$ hrs. \\
\hline $\begin{array}{c}\text { Includes Primi and } \\
\text { Multigravida No (\%) }\end{array}$ & $360(56.25)$ & $227(35.46)$ & $53(8.28)$ & $2(0.31)$ \\
\hline
\end{tabular}

Table 3. Average rate of cervical dilation (cm/hr.) between $4-6 \mathrm{~cm}$ and $6-10 \mathrm{~cm}$ cervical dilation in the study group.

\begin{tabular}{ccc}
\hline Average rate of dilatation $(\mathrm{cm} / \mathrm{hr})$. & $4-6 \mathrm{~cm}$ & $6-10 \mathrm{~cm}$ \\
\hline Mean & 1.71 & 3.14 \\
SD & 1.35 & 1.64 \\
Total (n) & 640 & 640 \\
\hline
\end{tabular}

t test-11.94; P value $<0.0001 ; \mathrm{CI}-1.19$ to 1.66

Table 4. Average rate of cervical dilation $(\mathrm{cm} / \mathrm{hr})$ between $4-6 \mathrm{~cm}$ and $6-10 \mathrm{~cm}$ cervical dilation in spontaneous and induced labour.

\begin{tabular}{ccc}
\hline $\begin{array}{c}\text { Average rate of dilatation of } \\
\text { cervix in primigravida and } \\
\text { multigravida }(\mathrm{cm} / \mathrm{hr} .)\end{array}$ & Between $4-6 \mathrm{~cm}$ dilatation & Between $6-10 \mathrm{~cm}$ dilatation \\
\hline Spontaneous $(\mathrm{n}=371)$ & $1.72(\mathrm{SD}=1.10)$ & $3.44(\mathrm{SD}=1.84)$ \\
Induced $(\mathrm{n}=269)$ & $1.68(\mathrm{SD}=1.63)$ & $2.69(\mathrm{SD}=1.18)$ \\
& $95 \% \mathrm{CI}=-0.252$ to 0.172 & $95 \% \mathrm{CI}=-1.001$ to -0.499 \\
$\mathrm{t}$ test $=-0.370$ & $\mathrm{t}$ test $=-5.86$ \\
$\mathrm{P}$ value $=0.711$ & $\mathrm{P}$ value $=<0.0001$ \\
\hline
\end{tabular}


When the rate of cervical dilation rate between $4-6 \mathrm{~cm}$ was compared we found $70.76 \%$ primigravida and $58.05 \%$ multigravida had a rate of $<1.5 \mathrm{~cm} / \mathrm{hr}$, while $29.23 \%$ primigravida and $41.94 \%$ multigravida had a rate of $>1.5 \mathrm{~cm} / \mathrm{hr}$ respectively. This difference was statistically significant $(P=0.0011)$. Hence in primigravida the cervix dilate at a slower rate between $4-6 \mathrm{~cm}$ cervical dilation in labour.

Comparing cervical dilation rate between $6-10 \mathrm{~cm}, 7.30 \%$ primigravida and $6.04 \%$ multi had a rate of $<1.5 \mathrm{~cm} / \mathrm{hr}$. while $92.69 \%$ primigravida and $83.44 \%$ multigravida had a rate of $>1.5 \mathrm{~cm} / \mathrm{hr}$. respectively. In this study we did not observe any difference in the rate cervical dilation amongst primi and multigravida between $6-10 \mathrm{~cm}$ cervical dilation. The observed results were not statistically significant $(\mathrm{P}=0.63)$ (Table 5).

Figure 1 and Figure 2 indicate cervix dilated at slower rate between $4-6 \mathrm{~cm}$ dilation as compared to between $6-10 \mathrm{~cm}$ cervical dilation. There was no significant difference between the rate of dilation between primigravida and multigravida between $6-10 \mathrm{~cm}$ of cervical dilatation as commented above.

In the study group majority of patients had lower rate of cervical dilation rate of $<2 \mathrm{~cm} / \mathrm{hr}$ between $4-6 \mathrm{~cm}$ cervical dilation and $>2 \mathrm{~cm} / \mathrm{hr}$. at $6 \mathrm{~cm}$ dilation (Figure 1 \& Figure 2). Slowest dilation rate of $<1 \mathrm{~cm} / \mathrm{hr}$. between $4-6 \mathrm{~cm}$ was observed in $20.2 \%$ primi and $12.8 \%$ multigravida.

Table 5. Rate of cervical dilation in primigravida and multigravida.

\begin{tabular}{ccccc}
\hline Dilatation & \multicolumn{2}{c}{$4-6 \mathrm{~cm}$} & \multicolumn{2}{c}{$6-10 \mathrm{~cm}$} \\
\hline & $<1.5 / \mathrm{hr}$. & $>1.5 / \mathrm{hr}$. & $<1.5 / \mathrm{hr}$. & $>1.5 / \mathrm{hr}$. \\
\hline Primigravida (342) No (\%) & $242(70.76)$ & $100(29.23)$ & $25(7.30 \%)$ & $317(92.69 \%)$ \\
Multi (298) No (\%) & $173(58.05 \%)$ & $125(41.94)$ & $18(6.04 \%)$ & $280(83.44 \%)$ \\
Chi-square value & \multicolumn{2}{c}{10.02} & 0.23 \\
P value & 0.0011 & 0.6300 \\
\hline
\end{tabular}

\section{Distribution of patients in different rates of cervical dilation observed $(\mathrm{cm} / \mathrm{hr}$.) between 4-6 cm cervical dilation}

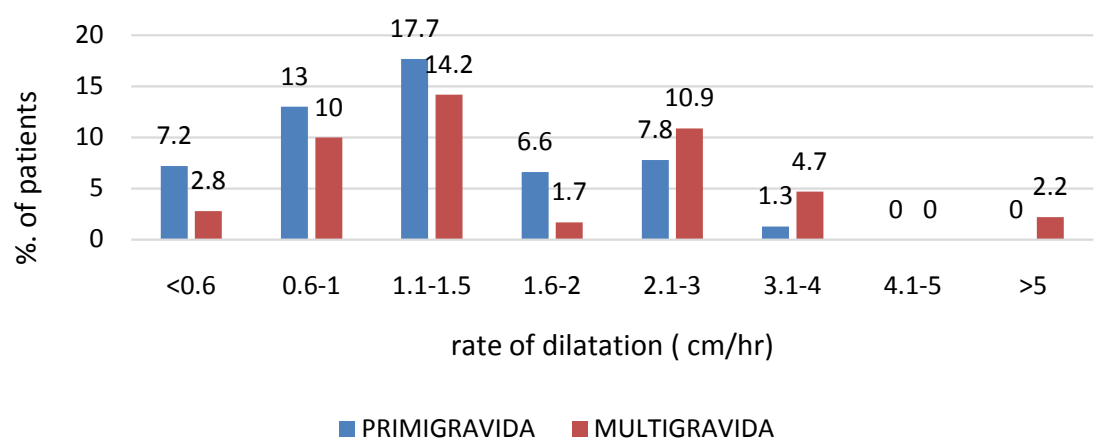

Figure 1. Distribution of patients in different rates of cervical dilation observed $(\mathrm{cm} / \mathrm{hr}$.) between 4 - $6 \mathrm{~cm}$ cervical dilation. 
Distribution of patients in different rates of cervical dilation observed $(\mathrm{cm} / \mathrm{hr}$.) between $6-10 \mathrm{~cm}$ cervical dilation

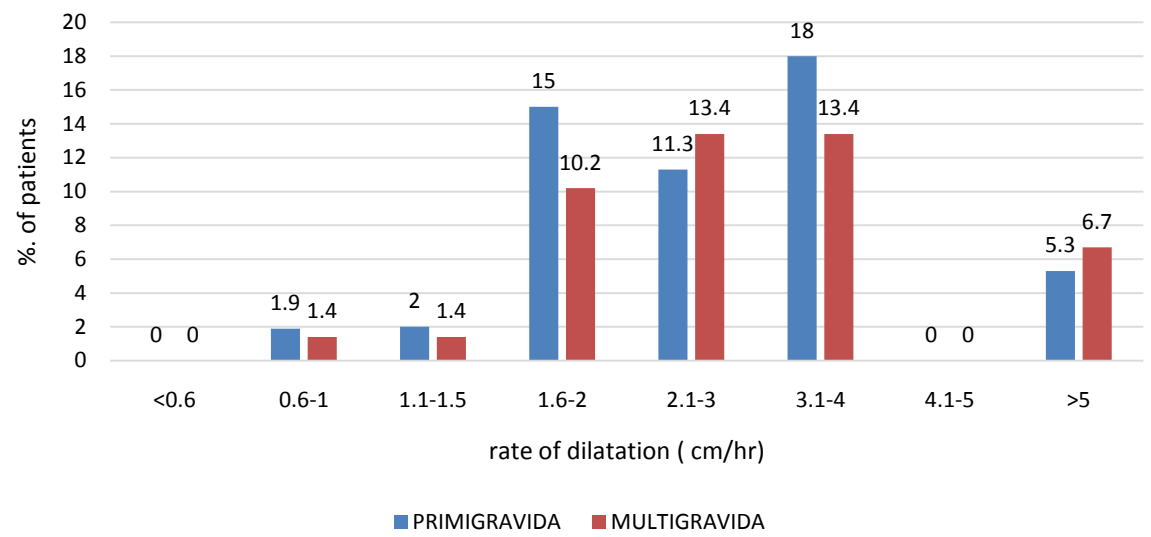

Figure 2. Distribution of patients in different rates of cervical dilation observed $(\mathrm{cm} / \mathrm{hr}$.) between $6-10 \mathrm{~cm}$ cervical dilation.

More rapid rate of cervical dilation up to $2-5 \mathrm{~cm} / \mathrm{hr}$. was noted in both primi \& multigravida only at $6 \mathrm{~cm}$ cervical dilation.

The perinatal outcome was good in all the cases in terms of APGAR score at 1 min and $5 \mathrm{~min}$. Only two neonates had APGAR of $<7$ at $1 \mathrm{~min}$ and they required NICU observation for 24 hours.

\section{Discussion}

We had studied a cohort of urban pregnant Indian women who were admitted after 34 weeks of pregnancy to determine the rate of cervical dilatation in the $1^{\text {st }}$ stage of labour progressing to normal delivery.

Since the 1950s management of the active phase of labour has been followed by the guidelines laid down by Friedman [13]. There are many published data available stating cervix dilates at slower rate in early labour. The risk of misdiagnosing dystocia and the overuse of labour accelerative interventions was found when Friedman's criteria of 1 or $>1 \mathrm{~cm} / \mathrm{hr}$. of cervical dilatation in active stage of labour was implemented. This may add unnecessary risk of intervention in mother and fetus. As per a study conducted by Jeremy O Neal in nulliparous women with spontaneous labour, the slowest normal linear cervical dilation is approximately $0.5 \mathrm{~cm} /$ hour [14]. As per the latest guidelines in normal labour there is no need to perform caesarean section in the latent phase of labour as it can be prolonged up to $54 \mathrm{hrs}$.

A systematic review conducted by O.T. Odalap described that in both nulliparous and parous women, labour accelerates (i.e. more than $1 \mathrm{~cm} /$ hour) between $5-6 \mathrm{~cm}$ of cervical dilatation [15]. Among the nulliparous women assessed, as compared to the accepted minimum threshold, 50\% of them had a much slower cervical dilatation rate of $0.5 \mathrm{~cm} /$ hour when cervical dilation was between $3-4 \mathrm{~cm}$. This rate of cervical dilation accelerated to a much faster rate of $2.04 \mathrm{~cm} /$ hour between $9-10 \mathrm{~cm}$ dilatation. 
According to Tilde B. Ostorg in Norway in an observational cohort study, nullipara with spontaneous labour had a shorter active phase of labour than in induced cases [16]. However, in induced cases of parous women, active phase of labour lasted for a short period of time before 6 hours while it was long after 6 hours of labor. Overall, this difference in parous women was insignificant.

We have observed significantly slower rates of cervical dilation in induced labour as compared to spontaneous labour between $6-10 \mathrm{~cm}$ of cervical dilation. $(\mathrm{P}<0.0001)$. But his difference was not significant between $4-6 \mathrm{~cm}$ cervical dilation $(\mathrm{P}-0.711)$.

Accurate latent phase duration calculations were not possible in our study group as we relied on history in some patients to determine the time of initiation of labour pains and calculate the duration of the latent phase. No cesarean sections were done in the latent phase. Hence only prolongation of latent phase should not be an indication to perform cesarean section. In this study $0.31 \%$ of patients in whom the latent phase was even prolonged up to $58 \mathrm{hrs}$., delivered vaginally.

In our study, the average rate of cervical dilation is almost double between 6 $10 \mathrm{~cm}$ cervical dilation as compared to between $4-6 \mathrm{~cm}$. In the study conducted by Yusuke Inde in Japanese females, it was observed that the fastest cervical changes occurred in active stage of labour at $6 \mathrm{~cm}$ in primigravida and $5 \mathrm{~cm}$ in multigravida. He concluded active labour may not start at $5 \mathrm{~cm}$ of cervical dilatation and at the beginning of active stage the cervix dilates at slower rate than previously described. These results may help in reducing the need for obstetric interventions during labour progression [17].

There was statistically significant difference in the rate of cervical dilation amongst primi and multigravida between $4-6 \mathrm{~cm}$ of cervical dilatation $(>1.5$ $\mathrm{cm} / \mathrm{hr}$.) but no such difference was seen between $6-10 \mathrm{~cm}$. It indicates cervix dilates at a slower rate in primi. Hence, we should be patient to perform Caesarean Section unless medically required.

Alexis C. in Thomas Jefferson University Hospital concluded from an RCT that the incidence of caesarean delivery decreased to around one half compared with usual guidelines when the length of labour extended in nulliparous women with singleton gestation with epidural anesthesia and prolonged second stage [18].

A study by Emily F Hamilton had suggested there was no significant relationship to hemorrhage or neonatal depression despite caesarean delivery carried out due to arrest of labour at or $>6 \mathrm{~cm}$ dilatation [19].

It is a matter of concern that a greater number of cesarean sections are being carried out before $5 \mathrm{~cm}$. dilation of cervix as Friedman's criteria of non-progressive labour and arrest disorders in labour are still being followed. Therefore it is time to redefine nonprogression and arrest disorder of labour based on large population studies of cervical dilatation rates in the 1 st stage of labour. This emphasizes the fact that active phase of labour is considered only at $6 \mathrm{~cm}$ dilation instead of $4 \mathrm{~cm}$ as was indicated by Friedman in 1954 . 
There is enough evidence that the labour progresses slower initially and rapid rate of cervical dilation is attained only at a higher cervical dilation. Women may take longer time to deliver without adverse perinatal outcome [20].

The present study has noted no abrupt change in the rate of cervical dilation in the 1st stage of labour, in fact gradual increase in the rate of cervical dilation was observed.

\section{Conclusions}

1) The active phase of labour i.e. rapid dilatation of cervix $(>1.5 \mathrm{~cm} / \mathrm{hr}]$ starts at $6 \mathrm{~cm}$ of dilation in the majority of the patients.

2) In multigravida, cervix dilates at faster rate before $6 \mathrm{~cm}$ while after $6 \mathrm{~cm}$ the rate of dilation observed is the same in comparison to primi.

3) In induced labour the active stage duration is longer i.e. there is significant difference between cervical dilation rates amongst induced and spontaneous labour after $6 \mathrm{~cm}$ cervical dilation.

4) Patients with a slowest cervical dilation rate of $<0.6 \mathrm{~cm} / \mathrm{hr}$. can deliver vaginally.

\section{Limitation of Our Study}

This is a single-center study. Patient profile and different intervention policies by healthcare providers from a particular center may differ from other center and hence the cesarean section rates may vary. Further studies are required to validate the fact as whether 5 or $6 \mathrm{~cm}$ cervical dilation should be taken as a point after which rapid cervical dilation is seen in majority.

\section{Conflicts of Interest}

The authors declare no conflicts of interest regarding the publication of this paper.

\section{References}

[1] Pitkin, R.M. (2003) Friedman E.A. Primigravid Labor: A Graphicostatistical Analysis. Obstet Gynecol 1955; 6: 567-89. Obstetrics \& Gynecology, 101, 216. https://doi.org/10.1016/S0029-7844(02)02398-0

[2] Philpott, R.H. (1972) Graphic Records in Labour. British Medical Journal, 4, 163-165. https://doi.org/10.1136/bmj.4.5833.163

[3] Rosenbloom, J.I., Stout, M.J., Tuuli, M.G., Woolfolk, C.L., López, J.D., Macones, G.A., et al. (2017) New Labor Management Guidelines and Changes in Cesarean Delivery Patterns. American Journal of Obstetrics and Gynecology, 217, 689.E1-689.E8. https://doi.org/10.1016/j.ajog.2017.10.007

[4] Zhang, J., Landy, H.J., Ware Branch, D., Burkman, R., Haberman, S., Gregory, K.D., et al. (2010) Contemporary Patterns of Spontaneous Labor with Normal Neonatal Outcomes. Obstetrics and Gynecology, 116, 1281-1287. https://doi.org/10.1097/AOG.0b013e3181fdef6e

[5] Middleton, P., Shepherd, E. and Crowther, C.A. (2018) Induction of Labour for 
Improving Birth Outcomes for Women at or Beyond Term. Cochrane Database of Systematic Reviews, 5, CD004945.

https://doi.org/10.1002/14651858.CD004945.pub4

[6] WHO (2011) Recommendation on the Use of Vaginal Misoprostol for Induction of Labour.

[7] Alfirevic, Z., Aflaifel N. and Weeks, A. (2014) Oral Misoprostol for Induction of Labour. Cochrane Database of Systematic Reviews, 2014, CD001338. https://doi.org/10.1002/14651858.CD001338.pub3

[8] Lapuente-Ocamica, O., Ugarte, L., Lopez-Picado, A., Sanchez-Refoyo, F., Lasa, I.L., Echevarria, O., et al. (2019) Efficacy and Safety of Administering Oral Misoprostol by Titration Compared to Vaginal Misoprostol and Dinoprostone for Cervical Ripening and Induction of Labour: Study Protocol for a Randomised Clinical Trial. BMC Pregnancy and Childbirth, 19, Article No. 14. https://doi.org/10.1186/s12884-018-2132-3

[9] MCPC. Induction and Augmentation of Labour. Health Education to Villages.

[10] Zhang, J., Branch, D.W., Ramirez, M.M., Laughon, S.K., Reddy, U., Hoffman, M., et al. (2011) Oxytocin Regimen for Labor Augmentation, Labor Progression, and Perinatal Outcomes. Obstetrics \& Gynecology, 118, 249-256.

https://doi.org/10.1097/AOG.0b013e3182220192

[11] Paul, A., Reena, Afzal, M., Bandyopadhyay, K. and Mishra, A. (2014) Labor Epidural Analgesia: Past, Present and Future. Indian Journal of Pain, 28, 71-81. https://doi.org/10.4103/0970-5333.132843

[12] Hanley, G.E., Munro, S., Greyson, D., Gross, M.M., Hundley, V., Spiby, H., et al. (2016) Diagnosing onset of Labor: A Systematic Review of Definitions in the Research Literature. BMC Pregnancy and Childbirth, 16, Article No. 71. https://doi.org/10.1186/s12884-016-0857-4

[13] Friedman, E.A. (1955) Primigravid Labor. Obstetrics \& Gynecology, 6, 567-589. https://doi.org/10.1097/00006250-195512000-00001

[14] Neal, J.L., Lowe, N.K., Patrick, T.E., Cabbage, L.A. and Corwin, E.J. (2010) What Is the Slowest-Yet-Normal Cervical Dilation Rate Among Nulliparous Women with Spontaneous Labor Onset? Journal of Obstetric, Gynecologic, \& Neonatal Nursing, 39, 361-369. https://doi.org/10.1111/j.1552-6909.2010.01154.x

[15] Oladapo, O.T., Diaz, V., Bonet, M., Abalos, E., Thwin, S.S., Souza, H., et al. (2018) Cervical Dilatation Patterns of "Low-Risk" Women with Spontaneous Labour and Normal Perinatal Outcomes: A Systematic Review. International Journal of Obstetrics and Gynaecology, 125, 944-954. https://doi.org/10.1111/1471-0528.14930

[16] Østborg, T.B., Romundstad, P.R. and Eggebø, T.M. (2017) Duration of the Active Phase of Labor in Spontaneous and Induced Labors. Acta Obstetricia et Gynecologica Scandinavica, 96, 120-127. https://doi.org/10.1111/aogs.13039

[17] Inde, Y., Nakai, A., Sekiguchi, A., Hayashi, M. and Takeshita, T. (2018) Cervical Dilatation Curves of Spontaneous Deliveries in Pregnant Japanese Females. International Journal of Medical Sciences, 15, 549-556. https://doi.org/10.7150/ijms.23505

[18] Gimovsky, A.C. and Berghella, V. (2016) Randomized Controlled Trial of Prolonged Second Stage: Extending the Time Limit vs Usual Guidelines. American Journal of Obstetrics and Gynecology, 214, 361.E1-361.E6. https://doi.org/10.1016/j.ajog.2015.12.042

[19] Hamilton, E.F., Warrick, P.A., Collins, K., Smith, S. and Garite, T.J. (2016) Assessing First-Stage Labor Progression and Its Relationship to Complications. American 
Journal of Obstetrics and Gynecology, 214, 358.E1-8.

[20] Abalos, E., Oladapo, O.T., Chamillard, M., Díaz, V., Pasquale, J., Bonet, M., et al. (2018) Duration of Spontaneous Labour in "Low-Risk" Women with "Normal" Perinatal Outcomes: A Systematic Review. European Journal of Obstetrics and Gynecology and Reproductive Biology, 223, 123-132.

https://doi.org/10.1016/j.ejogrb.2018.02.026 